\title{
On the interpretation of spectral-energy correlations in long gamma-ray bursts
}

\author{
L. Nava ${ }^{1,2}$, G. Ghisellini ${ }^{1}$, G. Ghirlanda ${ }^{1}$, F. Tavecchio ${ }^{1}$, and C. Firmani ${ }^{1,3}$ \\ 1 Osservatorio Astronomico di Brera, via Bianchi 46, 23807 Merate, Italy \\ e-mail: gabriele@merate.mi .astro.it \\ 2 Univ. di Milano-Bicocca, P.za della Scienza 3, 20126 Milano, Italy \\ ${ }^{3}$ Instituto de Astronomía, U.N.A.M., A.P. 70-264, 04510 México, D.F., México
}

Received 15 September 2005 / Accepted 6 November 2005

ABSTRACT

Context. Recently, Liang \& Zhang found a tight correlation involving only observable quantities, namely the isotropic emitted energy $E_{\gamma, \text { iso }}$, the energy of the peak of the prompt spectrum $E_{\mathrm{p}}^{\prime}$, and the jet break time $t_{\mathrm{j}}^{\prime}$ of Gamma Ray Bursts. This phenomenological correlation can have a first explanation in the framework of jetted fireballs, whose semiaperture angle $\theta_{\mathrm{j}}$ is measured by the jet break time $t_{\mathrm{j}}^{\prime}$. By correcting $E_{\gamma, \text { iso }}$ for the angle $\theta_{\mathrm{j}}$ one obtains the so-called Ghirlanda correlation, linking the collimation-corrected energy $E_{\gamma}$ and $E_{\mathrm{p}}^{\prime}$.

Aims. There are two ways to derive $\theta_{\mathrm{j}}$ from $t_{\mathrm{j}}^{\prime}$ in the "standard" scenario, corresponding to a homogeneous or to a wind-like circumburst medium. We compute and compare the $E_{\mathrm{p}}^{\prime}-E_{\gamma}$ correlations derived in these two conditions and study the consistency of these model-dependent correlations with the empirical Liang \& Zhang correlation. We consider the difference between the observed correlations and the ones in the comoving frame.

Methods. We study $18 \mathrm{GRBs}$ with firmly measured $z, E_{\text {peak }}$ and $t_{\text {break }}$ and discuss the differences with previously published samples. We compute the correlations accounting for the errors on all the relevant quantities.

Results. We show that the Ghirlanda correlation with a wind-like circumburst medium is as tight as (if not tighter) than the Ghirlanda correlation for a homogeneous medium. These two Ghirlanda correlations are both consistent with the phenomenological Liang \& Zhang relation. The wind-like Ghirlanda relation, which is linear, remains linear also in the comoving frame, independently of the distribution of bulk Lorentz factors. Instead, in the homogeneous density case, one is forced to assume the existence of a strict relation between the bulk Lorentz factor and the total energy, which in turn places constraints on the radiation mechanisms of the prompt emission. The wind-like Ghirlanda correlation, being linear, corresponds to different bursts having the same number of photons.

Key words. gamma rays: bursts - radiation mechanisms: non-thermal - X-rays: general

\section{Introduction}

The correlation between the collimation-corrected emitted energy in the prompt phase of GRBs $\left(E_{\gamma}\right)$ and the peak energy of the $v E_{v}$ prompt spectrum $\left(E_{\mathrm{p}}\right)$, the so-called "Ghirlanda" relation, is a fundamental tool for the cosmological use of GRBs.

Its importance calls for a robust and convincing explanation. Possible ideas and interpretations have been proposed, but require some ad hoc assumptions: different authors (Eichler \& Levinson 2004; Levinson \& Eichler 2005; Yamazaki et al. 2004; Toma et al. 2005) have underlined the importance of viewing angle effects, assuming different geometries of the fireball (annular or patchy); Rees \& Meszaros (2005) pointed out that a strict relation between total energy and typical peak frequency can be understood in a easier way if the underlying emission process is thermal, therefore suggesting that dissipative processes in the photosphere of the fireball can increase the thermal (black-body like) photon content of the fireball itself.
The collimation-corrected energy $E_{\gamma}$ is derived by multiplying the isotropic emitted energy $E_{\gamma, \text { iso }}$ by the factor (1 $\cos \theta_{\mathrm{j}}$ ), where $\theta_{\mathrm{j}}$ is the semiaperture angle of the jet. To derive it, one must assume the uniform jet model and the density profile of the circumburst medium (e.g. homogeneous or wind-like).

The Ghirlanda correlation has been derived for a homogeneous density (i.e. $n$ constant) and there are a few critical points concerning its derivation which are now under discussion.

Firstly, in this scenario the jet opening angle is (Sari et al. 1999)

$\theta_{\mathrm{j}}=0.161\left(\frac{t_{\mathrm{j}, \mathrm{d}}}{1+z}\right)^{3 / 8}\left(\frac{n \eta_{\gamma}}{E_{\gamma, \text { iso }, 52}}\right)^{1 / 8}$

where $z$ is the redshift, $\eta_{\gamma}$ is the radiative efficiency and $t_{\mathrm{j}, \mathrm{d}}$ is the achromatic break time, measured in days, of the afterglow 
lightcurve ${ }^{1}$. The efficiency $\eta_{\gamma}$ relates the isotropic kinetic energy of the fireball after the prompt phase, $E_{\mathrm{k}, \text { iso }}$, to the prompt emitted energy $E_{\gamma, \text { iso }}$, through $E_{\mathrm{k} \text {,iso }}=E_{\gamma, \text { iso }} / \eta_{\gamma}$. This implicitly assumes that $\eta_{\gamma} \ll 1$ otherwise the remaining kinetic energy after the prompt emission is instead $E_{\mathrm{k} \text {,iso }}=E_{\gamma \text {,iso }}\left(1-\eta_{\gamma}\right) / \eta_{\gamma}$. This efficiency, in principle, could be different from burst to burst, but in the absence of any hint of how its value changes as a function of other properties of the bursts and favoured by its low power in Eq. (1), one assumes a constant value for all bursts, i.e. $\eta_{\gamma}=0.2$ (after its first use by Frail et al. 2001, following the estimate of this parameter in GRB 970508).

Secondly, the density of the circumburst medium $n$ can in principle be estimated through accurate fits to the lightcurves at different frequencies (or, equivalently, fitting the spectral energy distributions (SEDs) at different times), but the fact that the emitted synchrotron spectrum is insensitive to $n$ in the regime of fast cooling makes the $n$ estimates somewhat uncertain. Furthermore, only for a minority of bursts we have enough data to constrain $n$ even in a rather poor way (see e.g. Panaitescu \& Kumar 2000, 2001). From this partial information, however, the estimated values of $n$ range from $\sim 0.1$ to $\sim 30 \mathrm{~cm}^{-3}$ with a clear preference for a homogeneous density scenario. Wind density profiles are acceptable and even preferred for few cases, in which however one cannot exclude the homogeneous density case. This led to the choice, made by Ghirlanda et al. (2004b, hereafter GGL04) to assume a homogeneous density scenario for all bursts and assign, to all cases in which $n$ was not estimated, a range of possible values of $n$, from 1 to $10 \mathrm{~cm}^{-3}$, to derive the value of $\theta_{\mathrm{j}}$ using Eq. (1), and, more importantly, the associated error. However, if GRBs are expected to originate from the death of very massive stars, the wind density profile appears as the most natural outcome of the final stages of the evolution of the burst progenitor.

These issues must caution us about the possible scatter of the points in the Ghirlanda correlation, which may be the result of bursts having a distribution (instead of a single value) of $n$ and $\eta_{\gamma}$, but on the other hand the extremely small scatter found suggests that, for the considered bursts, these values are indeed clustered over a small range.

These concerns (i.e. a possible distribution of $n$ and $\eta_{\gamma}$ values) have been overcome by the finding, by Liang \& Zhang (2005, hereafter LZ05), of a phenomenological and modelindependent correlation between $E_{\gamma, \text { iso }}, E_{\mathrm{p}}^{\prime}$ and $t_{\mathrm{j}}^{\prime}$. By considering 15 GRBs, and a flat Universe cosmology with $\Omega_{\mathrm{M}}=0.28$ and $h_{0}=0.713$, the correlation found by LZ05 takes the form:

$E_{\gamma, \text { iso }, 52}=(0.85 \pm 0.21)\left(\frac{E_{\mathrm{p}}^{\prime}}{100 \mathrm{keV}}\right)^{1.94 \pm 0.17} t_{\mathrm{j}, \mathrm{d}}^{-1.24 \pm 0.23}$

where primed quantities are calculated in the rest frame of the GRB, i.e. $t^{\prime}=t /(1+z)$ and $E_{\mathrm{p}}^{\prime}=E_{\mathrm{p}}(1+z)$. The scatter of the data points around this correlation is small enough to enable LZ05 to use it to find constraints on the cosmological parameters.

The main aim of the present paper is to discuss the steps we think necessary in order to deepen our understanding of the spectral/energy correlations in GRB, even if we do not

\footnotetext{
${ }^{1}$ Here we adopt the notation $Q=10^{x} Q_{x}$, and use cgs units unless otherwise noted.
}

claim to arrive to a complete or satisfactory interpretation. The Ghirlanda correlation (although derived earlier than the LZ05 relation) should be considered as a first step towards the explanation of the purely phenomenological LZ05 relation.

The first step is to demonstrate that the LZ05 correlation is equivalent to the Ghirlanda correlation. Secondly, we explore what happens to the Ghirlanda correlation if, instead of an homogeneous medium, we assume that the density is distributed with a $r^{-2}$ wind profile. We demonstrate that the LZ05 is again consistent with this new Ghirlanda-wind correlation.

We then have not one, but two possibilities to relate $E_{\mathrm{p}}$ and $E_{\gamma}$, both consistent with the LZ05 correlation. We argue that the new (wind-like) Ghirlanda correlation cannot be easily discarded on the basis of the fit to the afterglow SEDs, which fit better the uniform density case. The present small sample of GRBs does not allow us to test the two possibilities, but we provide one test to do so when estimates of the initial bulk Lorentz factor become available.

We then discuss a third step, pointing out that the observed $E_{\mathrm{p}}$ and $E_{\gamma}$ are affected by the relativistic motion of the fireball. In the simplest and standard scenario, which assumes that the observer's line of sight is within the jet opening angle and that the jet is homogeneous, $E_{\mathrm{p}}$ and $E_{\gamma}$ are both boosted by a factor of $\sim 2 \Gamma$, where $\Gamma$ is the bulk Lorentz factor of the fireball. Therefore what we see is an apparent correlation. Imposing that some correlation (even different from the observed one) exists in the comoving frame allows us to constrain both the dynamics and the emission process of bursts.

We report the updated tables for the relevant parameters used in deriving all the results and correlations discussed in the present paper. We discuss case-by-case the differences and changes of these quantities with respect to previously published papers.

\section{The sample}

LZ05 found their correlation (Eq. (2)) by using 15 bursts. Most of them are the same bursts as used by GGL04, but there are some differences in the reported parameters for the common GRBs. Also, since the publication of GGL04 some (primarily spectral) parameters have been updated (as a consequence of a refined analysis) and published in the literature. Also, other bursts have been detected recently, which bring the total number of GRBs with "useful" data (i.e. $z, E_{\mathrm{p}}$ and $t_{\mathrm{j}}$, excluding upper/lower limits) to 18 (as of September 2005). Our sample includes only those bursts with secure measurements of $z, E_{\text {peak }}, t_{\mathrm{j}}$. Consistency checks of the upper/lower limits can be performed (as shown in GGL04). For these reasons we present in Table 1 our sample of 18 GRBs with the relevant input data and corresponding references which represents the most updated collection of published parameters relevant for our analysis. We detail in the following all the relevant differences of this table with respect to those reported in GGL04 and LZ05.

Table 1 contains all GRBs reported by LZ05 with the exception of GRB 021211, and with the addition of 4 GRBs (GRB 970828, GRB 990705, GRB 041006, GRB 050525). Our exclusion of GRB 021211 is motivated by the extremely uncertain determination of $t_{\mathrm{j}}$ due to the scarcity of afterglow data 
Table 1. Input parameters for the bursts of our sample. $\alpha$ and $\beta$ are the photon spectral indices of the prompt emission spectrum and $E_{\mathrm{p}}$ represents the (observed) peak energy of the $v F_{v}$ spectrum. When the errors are not given in the original reference, we assumed an average error (values in square brackets), otherwise we list the originally reported error (in round brackets). ${ }^{a}$ References are given in order for the redshift ( $z$ ) and for the spectral parameters $(\alpha, \beta$, fluence and its energy interval): 1 Djorgovsky et al. (2001); 2 Jimenez et al. (2001); 3 Djorgovsky et al. (1998); 4 Hjorth et al. (1999); 5 Amati et al. (2002); 6 Vreeswijk et al. (2001); 7 Amati et al. (2000); 8 Vreeswijk et al. (1999); 9 Amati (2004); 10 Hjorth et al. (2003); 11 Atteia et al. (2005); 12 Price et al. (2003); 13 Barth et al. (2003); 14 Barraud et al. (2003); 15 Moller et al. (2002); 16 Sakamoto et al. (2005); 17 Greiner et al. (2003a); 18 Rol et al. (2003); 19 Greiner et al. (2003b); 20 Vanderspek et al. (2004); 21 Weidinger et al. (2003); 22 http://space.mit.edu/HETE/Bursts/); 23 Foley et al. (2005); 24 Blustin et al. (2005); ${ }^{b}$ References are given in order for the observed jet break time $t_{\mathrm{j}}$ and for the density $n$ when present: 25 Bloom et al. (2003); 26 Kulkarni et al. (1999); 27 Israel et al. (1999); 28 Bjornsson et al. (2001); 29 Jakobsson et al. (2003); 30 Berger et al. (2002); 31 Holland et al. (2003); 32 Klose et al. (2004); 33 Andersen et al. (2003); 34 Berger et al. (2003); 35 Jakobsson et al. (2004); 36 Panaitescu \& Kumar (2002); 37 Stanek et al. (2005); 38 Frail et al. (2005); 39 Blustin et al. (2005).

\begin{tabular}{lllllllllll}
\hline \hline GRB & $z$ & $\alpha$ & $\beta$ & $\begin{array}{l}\text { Fluence } \\
\mathrm{erg} / \mathrm{cm}^{2}\end{array}$ & $\begin{array}{l}\text { Range } \\
\mathrm{keV}\end{array}$ & $\begin{array}{l}E_{\mathrm{p}} \\
\mathrm{keV}\end{array}$ & $\begin{array}{l}\operatorname{Ref}^{a} \\
t_{\mathrm{j}} \\
\text { days }\end{array}$ & $\begin{array}{l}n \\
\mathrm{~cm}^{-3}\end{array}$ \\
\hline 970828 & 0.958 & $-0.70[0.08]$ & $-2.07[0.37]$ & $9.6 \mathrm{e}-5[0.9]$ & $20-2000$ & $298[60]$ & 1,2 & $2.2(0.4)$ & $3.0[2.76]$ & $25, \ldots$ \\
980703 & 0.966 & $-1.31[0.14]$ & $-2.40[0.26]$ & $2.26 \mathrm{e}-5[0.23]$ & $20-2000$ & $254[51]$ & 3,2 & $3.4(0.5)$ & $28(10)$ & 25,25 \\
990123 & 1.600 & $-0.89(0.08)$ & $-2.45(0.97)$ & $3.0 \mathrm{e}-4(0.4)$ & $40-700$ & $781(62)$ & 4,5 & $2.04(0.46)$ & $3.0[2.76]$ & $26, \ldots$ \\
990510 & 1.619 & $-1.23(0.05)$ & $-2.7(0.4)$ & $1.9 \mathrm{e}-5(0.2)$ & $40-700$ & $161(16)$ & 6,5 & $1.6(0.2)$ & $0.29(0.13)$ & 27,36 \\
990705 & 0.843 & $-1.05(0.21)$ & $-2.2(0.1)$ & $7.5 \mathrm{e}-5(0.8)$ & $40-700$ & $189(15)$ & 7,5 & $1.0(0.2)$ & $3.0[2.76]$ & $25, \ldots$ \\
990712 & 0.433 & $-1.88(0.07)$ & $-2.48(0.56)$ & $6.5 \mathrm{e}-6(0.3)$ & $40-700$ & $65(11)$ & 6,5 & $1.6(0.2)$ & $3.0[2.76]$ & $28, \ldots$ \\
991216 & 1.02 & $-1.23[0.13]$ & $-2.18[0.39]$ & $1.9 \mathrm{e}-4[0.2]$ & $20-2000$ & $318[64]$ & 8,2 & $1.2(0.4)$ & $4.7(3.5)$ & 25,36 \\
011211 & 2.140 & $-0.84(0.09)$ & $\ldots$ & $2.6 \mathrm{e}-6[0.3]$ & $40-700$ & $59(8)$ & 9,9 & $1.56[0.16]$ & $3.0[2.76]$ & $29, \ldots$ \\
020124 & 3.198 & $-0.87(0.17)$ & $-2.6(0.65)$ & $8.1 \mathrm{e}-6(0.9)$ & $2-400$ & $93(27)$ & 10,11 & $3.0[0.4]$ & $3.0[2.76]$ & $30, \ldots$ \\
020405 & 0.695 & $-0.0(0.25)$ & $-1.87(0.23)$ & $7.4 \mathrm{e}-5[0.7]$ & $15-2000$ & $364(101)$ & 12,12 & $1.67(0.52)$ & $3.0[2.76]$ & $12, \ldots$ \\
020813 & 1.255 & $-1.05[0.11]$ & $\ldots$ & $1.0 \mathrm{e}-4[0.1]$ & $30-400$ & $212[42]$ & 13,14 & $0.43(0.06)$ & $3.0[2.76]$ & $25, \ldots$ \\
021004 & 2.335 & $-1.0(0.2)$ & $\ldots$ & $2.6 \mathrm{e}-6(0.6)$ & $2-400$ & $80(35)$ & 15,16 & $4.74[0.5]$ & $3.0[2.76]$ & $31, \ldots$ \\
030226 & 1.986 & $-0.9(0.2)$ & $\ldots$ & $5.6 \mathrm{e}-6(0.6)$ & $2-400$ & $97(21)$ & 17,16 & $1.04(0.12)$ & $3.0[2.76]$ & $32, \ldots$ \\
030328 & 1.520 & $-1.14(0.03)$ & $-2.1(0.3)$ & $3.7 \mathrm{e}-5(0.14)$ & $2-400$ & $130(14)$ & 18,16 & $0.8[0.1]$ & $3.0[2.76]$ & $33, \ldots$ \\
030329 & 0.169 & $-1.32(0.02)$ & $-2.44(0.08)$ & $1.2 \mathrm{e}-4[0.12]$ & $30-400$ & $70(2)$ & 19,20 & $0.5(0.1)$ & $2.2[0.80]$ & 34,38 \\
030429 & 2.656 & $-1.1(0.3)$ & $\ldots$ & $8.5 \mathrm{e}-7(1.4)$ & $2-400$ & $35(10)$ & 21,16 & $1.77(1.0)$ & $3.0[2.76]$ & $35, \ldots$ \\
041006 & 0.716 & $-1.37[0.14]$ & $\ldots$ & $2.0 \mathrm{e}-5[0.2]$ & $25-100$ & $63[13]$ & 22,22 & $0.16(0.04)$ & $3.0[2.76]$ & $37, \ldots$ \\
050525 & 0.606 & $-0.99(0.11)$ & $\ldots$ & $2.01 \mathrm{e}-5(0.05)$ & $15-350$ & $79(3.5)$ & 23,24 & $0.28(0.12)$ & $3.0[2.76]$ & $39, \ldots$ \\
\hline
\end{tabular}

and to the likely "contamination" by a supernova (Della Valle et al. 2003). The value of $t_{\mathrm{j}}$ reported by LZ05 (1.4 day, see also Holland et al. 2004) should be considered only as a lower limit to $t_{\mathrm{j}}$ (as assumed in GGL04).

For GRBs listed in Table 1 that are also present in LZ04 we have the following (minor) differences:

- GRB 011211: the value of $t_{\mathrm{j}}$ reported by Jakobsson et al. (2003) is $t_{\mathrm{j}}=1.56 \pm 0.02$ days. This has been derived by fitting the light curve with a broken power law, which very likely severely underestimates the error on this quantity. Note that the $\chi^{2}$ of the original fit is large. Differently from LZ05, who used the above error, we have set the error to $10 \%$ (i.e. the average error on the observed jet break time) of the value of $t_{\mathrm{j}}$.

- GRB 020124: the spectral parameters have been updated by Atteia et al. (2005) with respect to the ones assumed by LZ05 from Sakamoto et al. (2005).

- GRB 020405: for the value of $E_{\mathrm{p}}$ listed in Table 1 we assume that in the original reference (Price et al. 2003), the authors presented the value $E_{0}$ of the Band spectrum. This corresponds to the e-folding energy of the exponential rollover of this model and it allows us to derive the peak energy as $E_{\mathrm{p}}=E_{0}(\alpha+2)$ (where $\alpha$ is the photon spectral index of the low energy power law component of the Band model).

Note also that if $\beta=-1.87 \pm 0.23$, as reported in Price et al. (2003), the spectral peak energy is unconstrained within the observational energy band. However, the reported uncertainty (0.23) makes the high energy spectral component consistent with $\beta<-2$. For this reason we assumed the lowest value, i.e. $\beta=-2.1$, to compute the isotropic equivalent energy of this burst.

- GRB 020813: we take the spectral parameters of this GRB from Barraud et al. (2003), while LZ05 use the values reported in Sakamoto et al. (2005). The reason of our choice is that, with the $\beta=-1.57 \pm 0.04$ reported by Sakamoto et al. (2005), one could not define the reported value of $E_{\mathrm{p}}=140 \pm 14 \mathrm{keV}$ (which instead requires at least $\beta<-2$ ). - GRB 021004: the value of $t_{\mathrm{j}}=4.74$ days comes from Holland et al. (2003), and it is the same (as well as the quoted reference) as LZ05. What is different is the error: Holland et al. (2003) report $t_{\mathrm{j}}=4.74_{-0.80}^{+0.14}$ days. 
Table 2. The rest frame peak energy $E_{\mathrm{p}}^{\prime}$ and the collimation corrected energy $E_{\gamma, \text { iso }}$ are calculated with the parameters reported in Table 1. The semiaperture angle $\theta_{\mathrm{j}}$ is calculated with Eq. (1) in the case of a homogeneous medium and the collimation corrected energy $E_{\gamma}$ is reported. We also report the values of $E_{\gamma, n=3}$ assuming $n=3 \mathrm{~cm}^{-3}$ for the 4 GRBs that have a different estimate of $n$ as reported in Table 1 . In the case of a wind medium the semiaperture angle $\theta_{\mathrm{j}, \mathrm{w}}$ (calculated by Eq. (6)) and the corresponding $E_{\gamma, \mathrm{w}}$ are reported. When the errors in $E_{\mathrm{p}}\left(E_{\gamma, \text { iso }}\right)$ are not given in the original reference, we assume an average error of $20 \%(11 \%)$ (values in square brackets), otherwise we list the originally reported error (in round brackets).

\begin{tabular}{llllllll}
\hline \hline GRB & $\begin{array}{l}E_{\mathrm{p}}^{\prime} \\
\mathrm{keV}\end{array}$ & $\begin{array}{l}E_{\gamma, \mathrm{iso}} \\
\mathrm{erg}\end{array}$ & $\begin{array}{l}\theta_{\mathrm{j}} \\
\mathrm{deg}\end{array}$ & $\begin{array}{l}E_{\gamma} \\
\mathrm{erg}\end{array}$ & $\begin{array}{l}E_{\gamma, n=3} \\
\mathrm{erg}\end{array}$ & $\begin{array}{l}\theta_{\mathrm{j}, \mathrm{w}} \\
\mathrm{deg}\end{array}$ & $\begin{array}{l}E_{\gamma, \mathrm{w}} \\
\mathrm{erg}\end{array}$ \\
\hline 970828 & $583[117]$ & $2.96 \mathrm{e} 53[0.35]$ & $5.91 \pm 0.79$ & $1.57 \mathrm{e} 51(0.46)$ & $\ldots$ & $3.40(0.19)$ & $5.21 \mathrm{e} 50(0.84)$ \\
980703 & $499[100]$ & $6.9 \mathrm{e} 52(0.82)$ & $11.02 \pm 0.80$ & $1.27 \mathrm{e} 51(0.24)$ & $7.29 \mathrm{e} 50(1.20)$ & $5.45(0.26)$ & $3.12 \mathrm{e} 50(0.48)$ \\
990123 & $2031(161)$ & $2.39 \mathrm{e} 54(0.28)$ & $3.98 \pm 0.57$ & $5.76 \mathrm{e} 51(1.78)$ & $\ldots$ & $1.84(0.12)$ & $1.24 \mathrm{e} 51(0.22)$ \\
990510 & $422(42)$ & $1.78 \mathrm{e} 53[0.19]$ & $3.74 \pm 0.28$ & $3.80 \mathrm{e} 50(0.69)$ & $6.80 \mathrm{e} 50(0.98)$ & $3.31(0.14)$ & $2.98 \mathrm{e} 50(0.40)$ \\
990705 & $348(28)$ & $1.82 \mathrm{e} 53(0.23)$ & $4.78 \pm 0.66$ & $6.33 \mathrm{e} 50(1.92)$ & $\ldots$ & $3.20(0.19)$ & $2.84 \mathrm{e} 50(0.50)$ \\
990712 & $93(16)$ & $6.72 \mathrm{e} 51(1.29)$ & $9.47 \pm 1.20$ & $9.16 \mathrm{e} 49(2.90)$ & $\ldots$ & $8.75(0.51)$ & $7.82 \mathrm{e} 49(1.76)$ \\
991216 & $642[129]$ & $6.75 \mathrm{e} 53[0.81]$ & $4.44 \pm 0.70$ & $2.03 \mathrm{e} 51(6.79)$ & $1.81 \mathrm{e} 51(0.50)$ & $2.36(0.21)$ & $5.72 \mathrm{e} 50(1.24)$ \\
011211 & $185(25)$ & $5.4 \mathrm{e} 52(0.6)$ & $5.38 \pm 0.66$ & $2.38 \mathrm{e} 50(0.64)$ & $\ldots$ & $4.24(0.16)$ & $1.48 \mathrm{e} 50(0.20)$ \\
020124 & $390[113]$ & $2.61 \mathrm{e} 53(0.18)$ & $5.07 \pm 0.64$ & $1.02 \mathrm{e} 51(0.27)$ & $\ldots$ & $3.13(0.12)$ & $3.90 \mathrm{e} 50(0.40)$ \\
020405 & $617[124]$ & $1.25 \mathrm{e} 53[0.13]$ & $6.27 \pm 1.03$ & $7.48 \mathrm{e} 50(2.58)$ & $\ldots$ & $4.08(0.34)$ & $3.17 \mathrm{e} 50(0.63)$ \\
020813 & $478[95]$ & $5.78 \mathrm{e} 53[0.58]$ & $2.8 \pm 0.36$ & $6.89 \mathrm{e} 50(1.88)$ & $\ldots$ & $1.85(0.08)$ & $3.00 \mathrm{e} 50(0.40)$ \\
021004 & $267(117)$ & $3.38 \mathrm{e} 52(0.78)$ & $8.47 \pm 1.06$ & $3.69 \mathrm{e} 50(1.25)$ & $\ldots$ & $6.20(0.40)$ & $1.98 \mathrm{e} 50(0.52)$ \\
030226 & $290[63]$ & $5.43 \mathrm{e} 52(0.68)$ & $4.71 \pm 0.58$ & $1.84 \mathrm{e} 50(0.51)$ & $\ldots$ & $3.88(0.17)$ & $1.24 \mathrm{e} 50(0.19)$ \\
030328 & $318[33]$ & $3.68 \mathrm{e} 53[0.37]$ & $3.58 \pm 0.45$ & $7.18 \mathrm{e} 50(1.93)$ & $\ldots$ & $2.35(0.10)$ & $3.09 \mathrm{e} 50(0.40)$ \\
030329 & $82(2)$ & $1.62 \mathrm{e} 52[0.16]$ & $5.69 \pm 0.50$ & $7.99 \mathrm{e} 49(1.62)$ & $8.63 \mathrm{e} 49(1.56)$ & $5.52(0.31)$ & $7.51 \mathrm{e} 49(1.13)$ \\
030429 & $128[26]$ & $1.42 \mathrm{e} 52(0.23)$ & $6.3 \pm 1.52$ & $8.57 \mathrm{e} 49(4.37)$ & $\ldots$ & $5.88(0.88)$ & $7.48 \mathrm{e} 49(2.55)$ \\
041006 & $108[22]$ & $6.92 \mathrm{e} 52[0.7]$ & $2.79 \pm 0.41$ & $8.18 \mathrm{e} 49(2.57)$ & $\ldots$ & $2.62(0.18)$ & $7.25 \mathrm{e} 49(1.23)$ \\
050525 & $135[8]$ & $2.3 \mathrm{e} 52[0.3]$ & $4.04 \pm 0.8$ & $5.73 \mathrm{e} 49(2.34)$ & $\ldots$ & $4.04(0.45)$ & $5.72 \mathrm{e} 49(1.40)$ \\
\hline
\end{tabular}

LZ05 take 0.14 days as the error in this quantity, while we take 0.5 days to better approximate the (asymmetric) error.

- GRB 030329: we have updated the spectral parameters of this burst according to Vanderspek et al. (2004), and these are somewhat different from those presented in the preprint version of the same article.

- GRB 050525: the prompt emission and the early afterglow detected by the Swift BAT, UVOT and XRT instruments have been analyzed by Blustin et al. (2005). These authors suggest the presence of a jet break in the very early afterglow lightcurve, i.e. $t_{\mathrm{j}}=0.16$ or 0.2 days according to two different model fits (the second value is found including also the data of Klotz et al. 2005, while the first value refers to the Swift data only). On the other hand, Mirabal et al. (2005) noted a jet break time at 0.4 days after trigger, based on a large collection of data (120 frames, still unpublished) taken with the 2.4 MDM meter telescope. Note also that the fit leading to $t_{\mathrm{j}}=0.2$ days assumes that the optical afterglow has a "jump" in its flux, but that thereafter continues to decay normally. In other words, this "discontinuity" is not treated as a "bump" in the lightcurve (as often seen in other bursts) which would have implied that the afterglow is composed of two contributions (the normal afterglow plus the re-brightening component). Fitting with this model would result in a larger value of $t_{\mathrm{j}}$. While waiting for a joint fit with all the data available, and also with different models, we decided to use $t_{\mathrm{j}}=0.28 \pm 0.12$ days: this value is intermediate between the Blustin et al. values and the Mirabal et al. value, with an error that encompasses all values.

In Table 2 we report the values of the rest frame peak energy $E_{\mathrm{p}}^{\prime}$

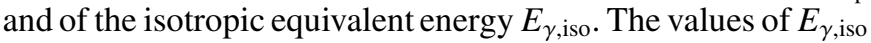
have been taken directly from Amati et al. (2002) in the case of GRBs detected by BeppoSax and listed in that paper, but we converted these values to our cosmology (i.e. we use $h=0.7$ while Amati et al. 2002 used $h=0.65$ ).

Tables 1 and 2 are the updated version of the tables presented in GGL04 which were composed of 15 "usable" GRBs. With respect to that paper, our present sample of 18 GRBs comprises 3 new GRBs (GRB 021004, GRB 041006 and GRB 050525) for which $z, E_{\mathrm{p}}$ and $t_{\mathrm{j}}$ have been published and some of the parameters have changed due to updates in the literature.

- GRB 991216: the value of the density $n=4.70_{-1.8}^{+6.8}$ was estimated by Panaitescu \& Kumar (2002). We have here assumed a symmetric error equal to the logarithmic average, and taken $n=4.7 \pm 3.5$. This differs slightly from what assumed in GGL04 $(n=4.7 \pm 2.3)$.

- GRB 011211: we changed the reference for the spectral parameters, which is Amati (2004), and not Amati et al. (2002) as given in GGL04. We recalculated the value of $E_{\gamma, \text { iso }}$ with $h=0.7$. 
- GRB 020124: we updated the spectral parameters, now taken from Atteia et al. (2005). The main difference concerns $E_{\mathrm{p}}=120 \mathrm{keV}$, instead of the value of $93 \mathrm{keV}$ reported in GGL04.

- GRB 030226 and GRB 030328: we now use the spectral parameters reported in Sakamoto et al. (2005) (instead of Atteia 2003) and the recalculated $E_{\gamma, \text { iso. }}$.

- GRB 030329: as mentioned above, we updated the spectral parameters according to the published paper by Vanderspek et al. (2004). The listed value of $n=2.2 \mathrm{~cm}^{-3}$ comes from Frail et al. (2005) (GGL04, instead, quoted $n=1 \mathrm{~cm}^{-3}$ ): the associated error encompasses the three possible values listed in Table 2 of Frail et al. (2005).

- GRB 030429: we updated the spectral parameters with Sakamoto et al. (2005) instead of those assumed in GGL04 which were taken from the Hete- 2 web page.

\section{The spectral-energy correlations}

With the updated sample of 18 GRBs reported in Table 1 we first refit the empirical LZ05 correlation among $E_{\gamma, \text { iso }}, E_{\mathrm{p}}^{\prime}$ and $t_{\mathrm{j}}^{\prime}$. We also give the updated version of the Ghirlanda correlation (GGL04 and Ghirlanda et al. 2005a) in the case of a homogeneous density profile. Finally we present the Ghirlanda correlation in the case of a wind density profile.

\subsection{The Liang-Zhang correlation revisited}

The method used by LZ05 to find the correlation between $E_{\gamma, \text { iso }}, E_{\mathrm{p}}^{\prime}$ and $t_{\mathrm{j}}^{\prime}$ is a multivariate linear regression. The significance of the multivariate regression is estimated through the Ftest and through the Spearman $r_{\mathrm{s}}$ coefficient between $\log E_{\gamma, \text { iso }}$ calculated with Eq. (2) and the same quantity directly calculated using

$E_{\gamma, \text { iso }}=\frac{4 \pi d_{L}^{2} S_{\gamma} k}{1+z}$

where $d_{L}$ is the luminosity distance, $S_{\gamma}$ the $\gamma$-ray fluence in the observed energy band, $k$ is the bolometric correction factor needed to find the energy emitted in a fixed energy range (here, $1-10000 \mathrm{keV}$ ) in the rest frame of the source.

We have used a different method that enables us to weight the multidimensional fit for the errors on the three independent variables $E_{\gamma, \text { iso }}, E_{\mathrm{p}}^{\prime}$ and $t_{\mathrm{j}}^{\prime}$. We have extended the procedure for the fit of a straight line to data with errors on two coordinates to a three dimensional space (Press et al. 1999), so that we use the $\chi^{2}$ statistics to find the best fit.

Assuming a $\Omega_{\mathrm{M}}=0.3$ and $h=\Omega_{\Lambda}=0.7$ cosmology, we find

$E_{\gamma, \text { iso }, 53}=(1.12 \pm 0.11)\left(\frac{E_{\mathrm{p}}^{\prime}}{295 \mathrm{keV}}\right)^{1.93 \pm 0.17}\left(\frac{t_{\mathrm{j}}^{\prime}}{0.51 \mathrm{~d}}\right)^{-1.08 \pm 0.17}$

with a reduced $\chi_{\mathrm{r}}^{2}=1.49$. The $3 \mathrm{D}$ plot of the data points in the $E_{\gamma, \text { iso }}, E_{\mathrm{p}}^{\prime}$ and $t_{\mathrm{j}}^{\prime}$ space and the best fit plane as defined by Eq. (4) are represented in Fig. 1. Similarly to what has been done in 2D (GGL04) we can define the scatter of the data points around the best fit plane through their distance computed perpendicular to

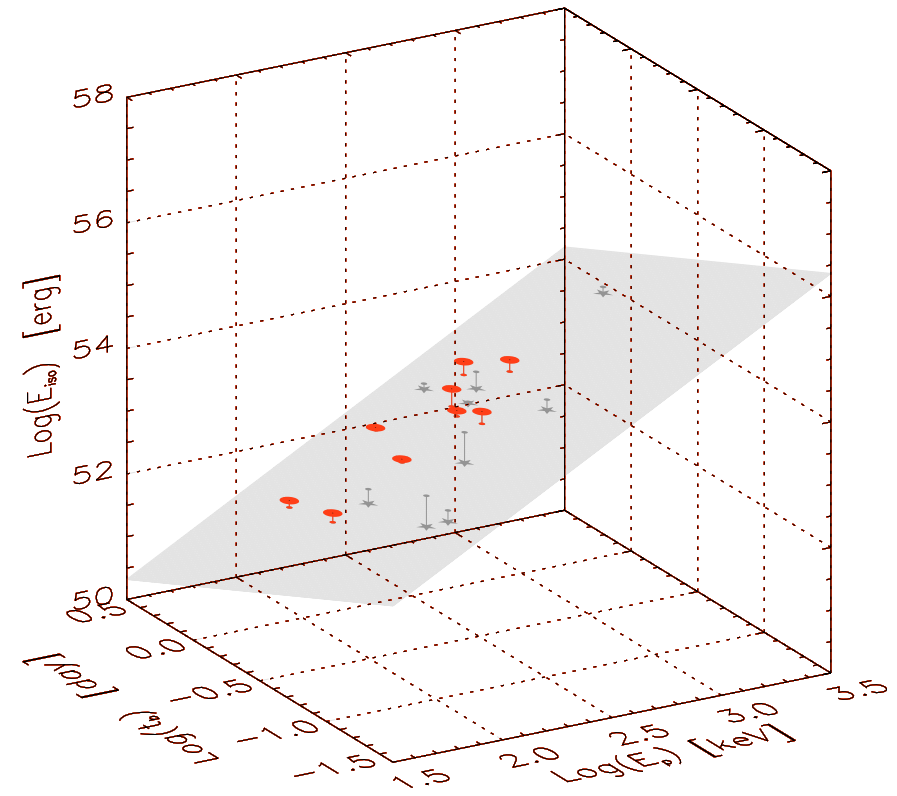

Fig. 1. Three dimensional representation of the $E_{\gamma, \text { iso }}\left(E_{\mathrm{p}}^{\prime}, t_{\mathrm{j}}^{\prime}\right)$ correlation. Data points are from Table 2. The plane is the best fit to the data points as represented by Eq. (4) which has a reduced $\chi_{\mathrm{r}}^{2}=1.49$. The red points lie above the best fit plane while the grey stars are below the plane. The height of each point with respect to the best fit plane is also shown.

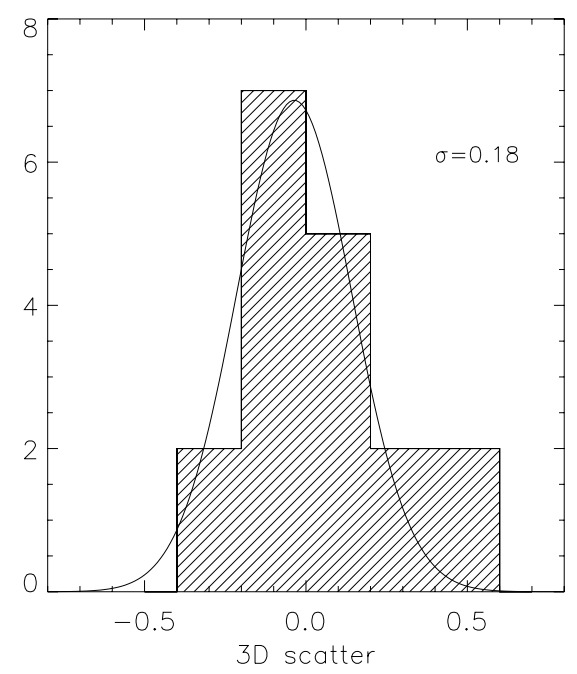

Fig. 2. Histogram of the scatter of the data points computed perpendicular to the best fit plane in the 3D space of Fig. 1. The solid line represents the Gaussian fit which has a $\sigma=0.18$.

this plane. The histogram of the scatter is reported in Fig. 2 and, when fitted with a Gaussian it has a $\sigma=0.18$.

Comparing Eq. (4) with the original result of LZ05 we obtain a value of the $t_{\mathrm{j}}^{\prime}$ exponent closer to unity, but still consistent with the value of LZ05 (which was also consistent with unity, due to the relatively larger uncertainty). As explained in the next section, a value equal to unity is crucial to make the LZ05 correlation and the Ghirlanda correlation mutually consistent.

We show in Fig. 3 the values of $E_{\gamma \text {,iso }}$ calculated with Eq. (3) as a function of $E_{\gamma \text {,iso }}$ calculated using the best fit of the correlation $E_{\gamma, \text { iso }}\left(E_{\mathrm{p}}^{\prime}, t_{\mathrm{j}}^{\prime}\right)$ found in our data and with our method, 


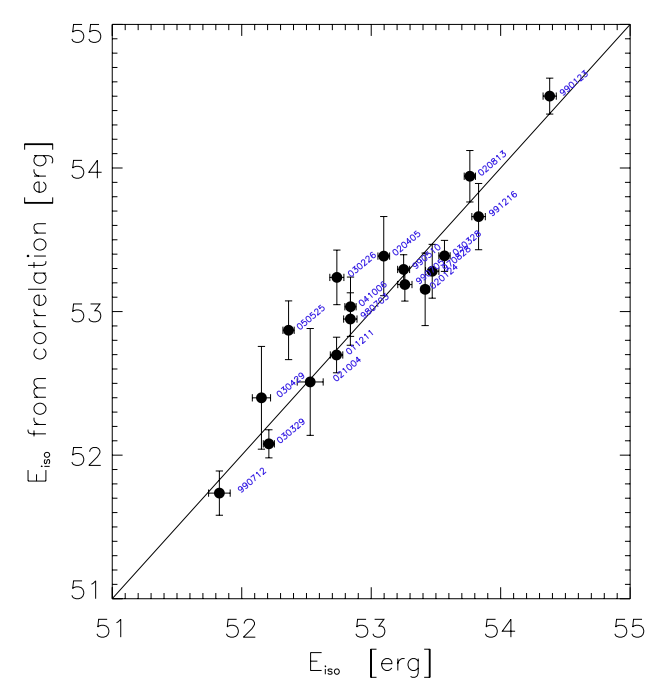

Fig. 3. $E_{\gamma, \text { iso }}$ calculated with the best fit multidimensional correlation $E_{\gamma, \text { iso }}\left(E_{\mathrm{p}}^{\prime}, t_{\mathrm{j}}^{\prime}\right)($ Eq. (4)) as a function of the same quantity calculated through Eq. (3).

and assuming a $\Omega_{\mathrm{M}}=0.3$ and $h=\Omega_{\Lambda}=0.7$ cosmology. As can be seen, there is a very good agreement.

It is also interesting, in view of the discussion of the following sections, to fit the $E_{\gamma, \text { iso }}, E_{\mathrm{p}}^{\prime}$ and $t_{\mathrm{j}}^{\prime}$ correlation by forcing the exponent of $t_{\mathrm{j}}^{\prime}$ to be -1 . Fixing it we find $E_{\gamma, \text { iso }} t_{\mathrm{j}}^{\prime} \propto E_{\mathrm{p}}^{1.91 \pm 0.1}$, with a reduced $\chi_{\mathrm{r}}^{2}=1.51$.

\subsection{The updated Ghirlanda correlation}

Using the same data listed in Table 1 we calculate the updated version of the Ghirlanda correlation. We find a Spearman correlation coefficient $r_{\mathrm{s}}=0.93$ with a chance probability $P=2.3 \times 10^{-8}$. We report in Fig. 4 the updated correlation with the 18 GRBs reported in Table 1.

We fitted this correlation with a power law model accounting for the errors on both variables, i.e. $E_{\mathrm{p}}^{\prime}$ and $E_{\gamma}$ (using the routine fitexy of Press et al. 1999). For $\Omega_{\mathrm{M}}=0.3, \Omega_{\Lambda}=h=0.7$ we find

$\left(\frac{E_{\mathrm{p}}^{\prime}}{100 \mathrm{keV}}\right)=(2.79 \pm 0.15)\left(\frac{E_{\gamma}}{2.72 \times 10^{50} \mathrm{erg}}\right)^{0.69 \pm 0.04}$

with a reduced $\chi_{\mathrm{r}}^{2}=1.4$ for 16 degrees of freedom. The errors on its slope and normalization are calculated in the "barycenter" of $E_{\mathrm{p}}^{\prime}$ and $E_{\gamma}$, where the slope and normalization errors are uncorrelated (Press et al. 1999). The simplest linear regression fit (i.e. without accounting for errors on the variables) gives a slope of 0.6 (dotted line in Fig. 4).

Figure 4 shows the correlation for the $18 \mathrm{GRBs}$ and its best fit represented by Eq. (5) (solid line). This updated correlation has a slope consistent with the original value found in GGL04. We also computed the scatter of the data points around this correlation (inset in Fig. 4). This scatter is defined as the distance in the $\log E_{\mathrm{p}}^{\prime}-\log E_{\gamma}$ plane of each data point from the best fit correlation. We find that if fitted with a Gaussian its standard deviation is $\sigma=0.1$, i.e. lower than the value originally found by GGL04.

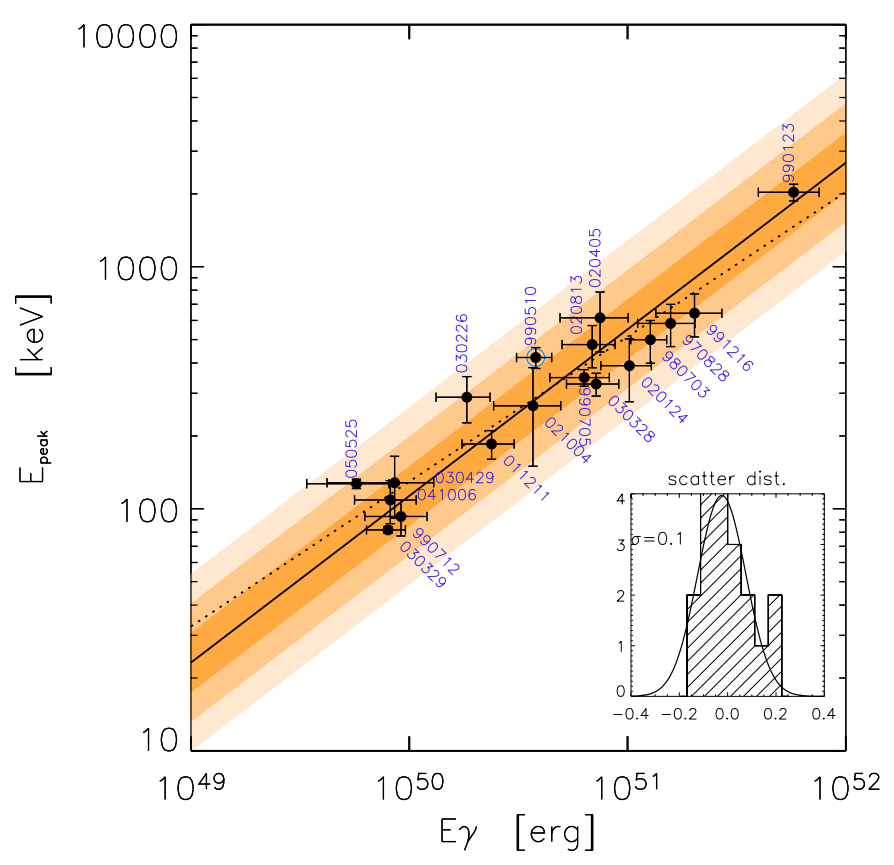

Fig. 4. The updated Ghirlanda correlation between the rest frame spectral peak energy $E_{\mathrm{p}}^{\prime}$ and the collimation corrected energy $E_{\gamma}$ as found with the 18 GRBs reported in Table 1 . The solid line represents the best fit power law model obtained accounting for the errors on both coordinates (Eq. (5)), which has a reduced $\chi_{\mathrm{r}}^{2}=1.4$ (16 d.o.f.) and a slope of $0.69 \pm 0.04$. We also show the fit obtained with the simplest linear regression, i.e. without accounting for the errors on the coordinates (dotted line, slope equal to 0.6 ). The circled point represents GRB 990510 which alone contributes $27 \%$ of the total $\chi^{2}$ of the fitted model. The names of the 18 GRBs are also reported. The shaded areas represent the regions corresponding to the 1,2 and $3 \sigma$ scatter around the best fit correlation. The inset reports the distribution (hatched histogram) of the scatter of the data points computed perpendicularly to the best correlation (solid line in main plot) and its Gaussian fit (solid line in the inset) which has a $\sigma=0.1$.

For completeness, we also computed the Amati correlation with the 18 GRBs reported in Table 1 . By weighting for the errors on $E_{\mathrm{p}}$ and $E_{\gamma, \text { iso }}$, we find a relatively poor fit with a reduced $\chi_{\mathrm{r}}^{2}=5.22$ and a best fit correlation $E_{\gamma, \text { iso }} \propto E_{\mathrm{p}}^{0.57 \pm 0.02}$.

\subsection{The Ghirlanda correlation in the case of a wind density profile}

If the external medium is distributed with an $r^{-2}$ density profile the semiaperture angle of the jet is related to the achromatic jet break through (Chevalier \& Li 2000):

$\theta_{\mathrm{j}, \mathrm{w}}=0.2016\left(\frac{t_{\mathrm{j}, \mathrm{d}}}{1+z}\right)^{1 / 4}\left(\frac{\eta_{\gamma} A_{*}}{E_{\gamma, \text { iso }, 52}}\right)^{1 / 4}$

where we assume $n(r)=A r^{-2}$ and $A_{*}$ is the value of $A(A=$ $\left.\dot{M}_{\mathrm{w}} /\left(4 \pi v_{\mathrm{w}}\right)=5 \times 10^{11} A_{*} \mathrm{~g} \mathrm{~cm}^{-1}\right)$ when setting the mass loss rate due to the wind $\dot{M}_{\mathrm{w}}=10^{-5} M_{\odot} \mathrm{yr}^{-1}$ and the wind velocity $v_{\mathrm{w}}=10^{3} \mathrm{~km} \mathrm{~s}^{-1}$, according to the Wolf-Rayet wind physical conditions.

In the wind case we use Eq. (6) to correct the isotropic energies $E_{\gamma, \text { iso }}$ by the factor $\left(1-\cos \theta_{\mathrm{j}, \mathrm{w}}\right)$. Given the few still 


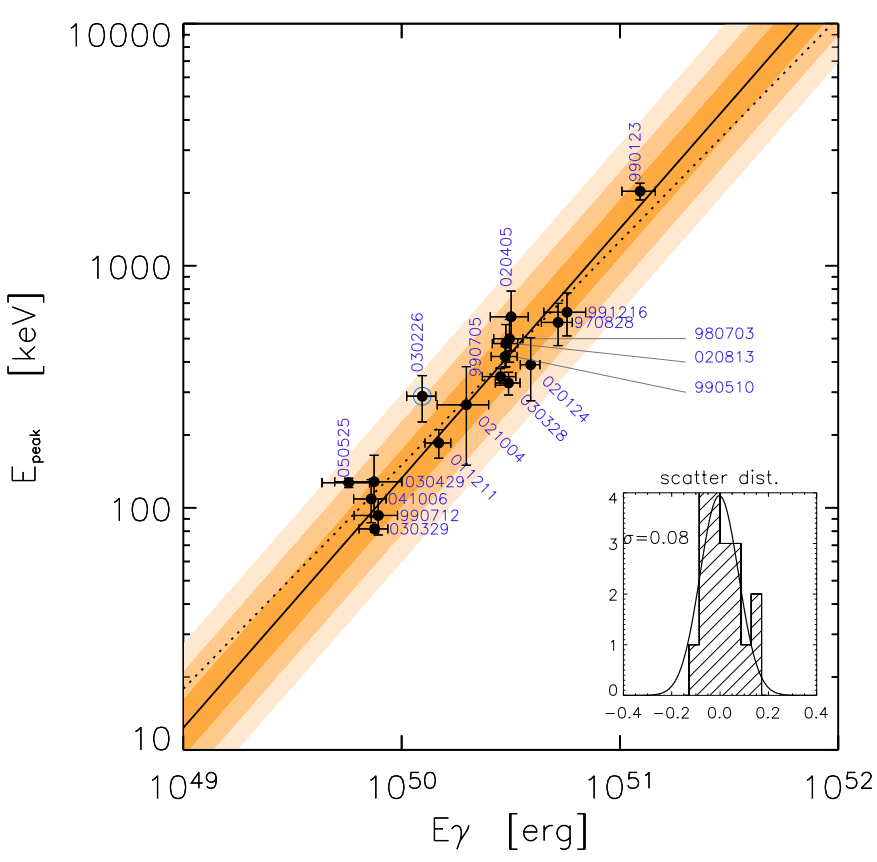

Fig. 5. The Ghirlanda correlation in the case of a wind profile of the external medium density as found with the 18 GRBs reported in Table 1 (Col. 5). The values of $E_{\gamma}$ are reported in Table 2. The solid line represents the best fit power law model obtained accounting for the errors on both coordinates (Eq. (7)) which has a reduced $\chi_{\mathrm{r}}^{2}=1.125$ (16 d.o.f.) and a slope of $1.03 \pm 0.06$. We also show the fit obtained with the simplest linear regression, i.e. without accounting for the errors on the coordinates (dotted line), which has a slope of 0.92 . The circled point represents GRB 030326 which provides the largest contribution $(23 \%)$ to the best fit reduced $\chi^{2}$. The shaded regions represent the 1,2 and $3 \sigma$ scatter around the best fit correlation. The names of the 18 GRBs are indicated. The inset reports the distribution (hatched histogram) of the scatter of the data points computed perpendicularly to the best correlation (solid line in the main plot) and its Gaussian fit (solid line in the inset) which has a $\sigma=0.08$.

uncertain estimates of the $A_{*}$ parameter, we assume a typical value (i.e. $A_{*}=1$ ) for all bursts neglecting for the moment the possible uncertainty on this parameter.

In the wind case we find a correlation between $E_{\mathrm{p}}^{\prime}$ and $E_{\gamma}$ with a Spearman rank correlation coefficient $r_{\mathrm{s}}=0.92(P=$ $6.9 \times 10^{-8}-$ see also Zeh et al. 2005). The fit with a power law model gives

$\frac{E_{\mathrm{p}}^{\prime}}{100 \mathrm{keV}}=(3.0 \pm 0.16)\left(\frac{E_{\gamma}}{2.2 \times 10^{50} \mathrm{erg}}\right)^{1.03 \pm 0.06}$

with a reduced $\chi_{\mathrm{r}}^{2}=1.13$ for 16 d.o.f. (see Fig. 5). Note that the exponent of this new relation is consistent with unity. The scatter of the points (insert of Fig. 5) around the best fit correlation is fitted by a Gaussian with $\sigma=0.08$.

Since we have no knowledge of the uncertainty associated with the $\eta A_{*}$ parameter entering in Eq. (6), we estimate that, for the assumed typical value $\eta A_{*}=0.2$, an error $\sigma_{\eta A_{*}} \leq 20 \%$ does not dominate the fit of the correlation (i.e. the reduced $\chi_{\mathrm{r}}^{2}$ is not much smaller than 1).

Since we have assumed that all the $A_{*}$ values are equal and have no errors, the resulting $\chi_{\mathrm{r}}^{2}$ of the wind case should be compared with the the case of homogeneous density assuming all

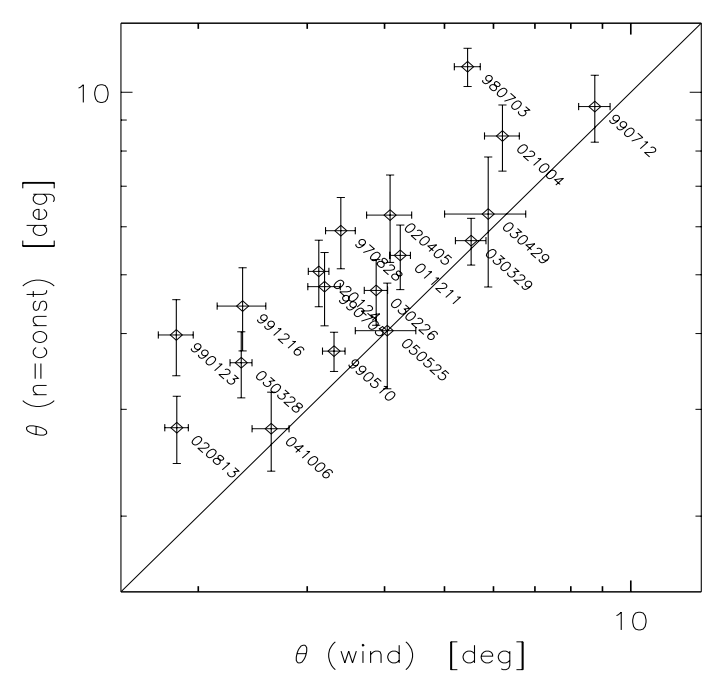

Fig. 6. Jet opening angles calculated for a homogeneous density profile $\left(\theta_{j}\right)$ and for a wind density profile $\left(\theta_{j, w}\right)$ for the 18 GRBs reported in Table 2 (Cols. 4 and 7 respectively).

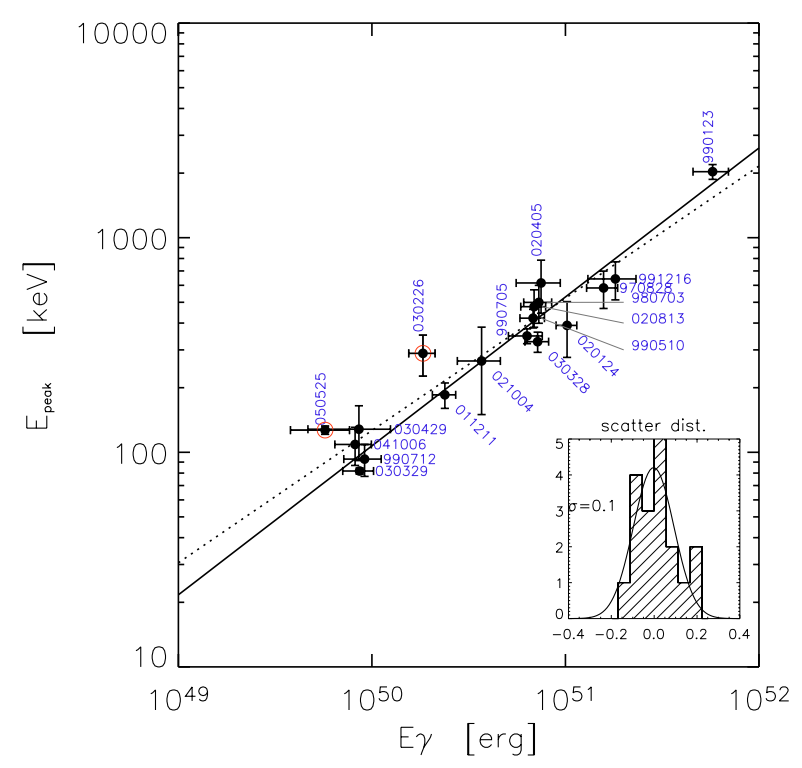

Fig. 7. The Ghirlanda correlation in the case of a homogeneous external medium assuming a universal value for the density, i.e. $n=3.0$ without uncertainty, for comparison with the wind case. The solid line represents the best fit power law model obtained accounting for the errors on both coordinates which has a reduced $\chi_{\mathrm{r}}^{2}=1.4$ (16 d.o.f.) and a slope of $0.69 \pm 0.04$.

the $n$ values equal (we set $n=3 \mathrm{~cm}^{-3}$ ) with no error (see Table 2). This case is shown in Fig. 7. In this case we obtain $\chi_{\mathrm{r}}^{2}=1.4$, to be compared with the $\chi_{\mathrm{r}}^{2}=1.125$ in the wind case. We conclude that the wind case gives a somewhat smaller $\chi_{\mathrm{r}}^{2}$ and a somewhat tighter (smaller scatter) and a steeper correlation than that found in the case of a homogeneous medium. Therefore, even if the wind density profile is not favored by the afterglow model, we cannot discard it on the basis of the Ghirlanda relation.

The jet opening angles calculated in the case of a homogeneous medium or in the case of a wind density profile for the sample of $18 \mathrm{GRBs}$ is reported in Fig. 6. The angle calculated 
in the wind case (Eq. (6)) is systematically smaller than in the homogeneous medium case (Eq. (1)).

\section{Consistency of the empirical correlation with the model dependent correlations}

In this section we demonstrate that the Ghirlanda correlation either assuming a homogeneous circumburst density or a wind density profile and the LZ05 correlations are mutually consistent. This allows us to make some interesting considerations on the scatter of the Ghirlanda correlations. The fact that the LZ05 and the Ghirlanda correlation are mutually consistent was pointed out by LZ05 (see also $\mathrm{Xu} 2005$ ) in the case of a homogeneous medium.

For the simple analytical demonstration, we consider a generic form of the LZ05 correlation, namely:

$E_{\gamma, \text { iso }} \propto E_{\mathrm{p}}^{\prime A} t_{\mathrm{j}}^{\prime B}$

\subsection{Homogeneous density}

Adopting the standard fireball scenario, assuming a uniform jet and a homogeneous circumburst density distribution, the relation between $t_{\mathrm{j}}^{\prime}$ and $\theta_{\mathrm{j}}$ is given by Eq. (1). Inserting it into Eq. (8) one obtains

$E_{\gamma, \text { iso }} \theta_{\mathrm{j}}^{2} \sim E_{\gamma} \propto E_{\mathrm{p}}^{\prime 3 A /(3-B)} \theta_{\mathrm{j}}^{6(B+1) /(3-B)}\left(n \eta_{\gamma}\right)^{-B /(3-B)}$

where we have used the small angle approximation (1 $\left.\cos \theta_{\mathrm{j}}\right) \propto \theta_{\mathrm{j}}^{2}$. Equation (9) relates five variables.

We have shown that the Ghirlanda correlation is characterized by a scatter $\sigma \sim 0.1$ (Sect. 3.2 and Fig. 4). If this scatter is entirely due to dispersion of the $n \eta_{\gamma}$ values (and very likely also to the errors of measurements on the observables) and not to $\theta_{\mathrm{j}}$, we derive the condition $B=-1$ and Eq. (9) reduces to a relation between $E_{\gamma}$ and $E_{\mathrm{p}}^{\prime}$. This value of $B$ is consistent with the value found from the fit of the LZ05 correlation (i.e. $B=-1.08 \pm 0.17$ ) with the $18 \mathrm{GRBs}$ of our sample. If the exponent $B=-1$, then the slope $g$ of the Ghirlanda correlation (i.e. $E_{\mathrm{p}}^{\prime} \propto E_{\gamma}^{g}$ ) is related to the exponent $A$ of the LZ05 correlation through $g=4 /(3 A)$.

On the other extreme, if the scatter of the Ghirlanda correlation is completely due to $\theta_{\mathrm{j}}$ (and to the errors of measurements on the observables) we may still derive a range of allowance for the parameter $B$. We computed the standard deviation $\sigma$ of the distribution of $\theta_{\mathrm{j}}^{6(B+1) /(3-B)}$ as a function of $B$. This is represented in Fig. 8 by the solid line. If we compare $\sigma$ with the scatter of the Ghirlanda correlation (in the homogeneous density case - solid horizontal line in Fig. 8) we find quite shallow constraints on $B \in(-1.3,-0.7)$.

Clearly, the intermediate case corresponds to both $n \eta_{\gamma}$ and $\theta_{\mathrm{j}}$ contributing to the scatter. If we consider the range of $B$ as found by fitting the LZ05 correlation (Eq. (4)), i.e. $B=-1.08 \pm 0.17$ (shaded region in Fig. 8) then the term $\theta_{\mathrm{j}}^{6(B+1) /(3-B)}$ can contribute at most $\sim 70 \%$ of the total scatter of the Ghirlanda correlation.

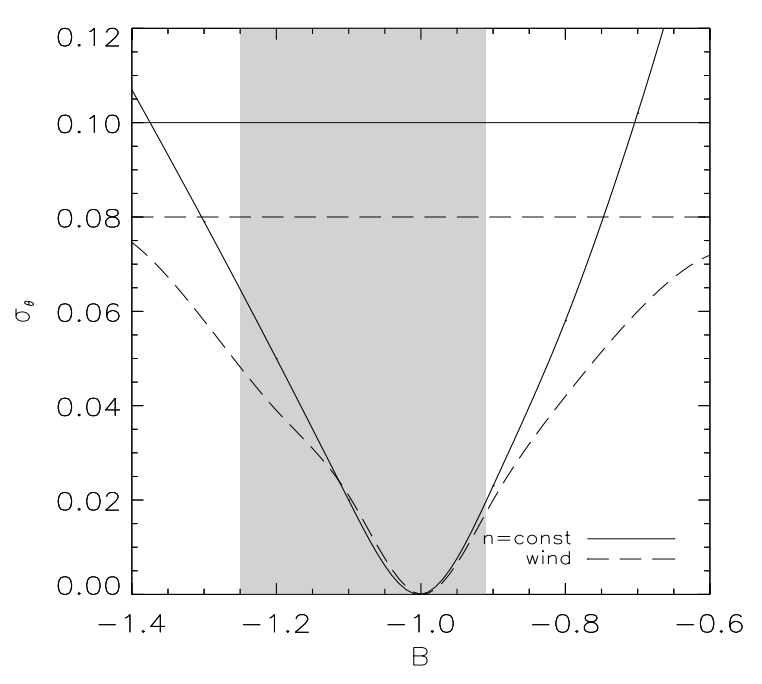

Fig. 8. Standard deviation of the distribution of $\theta^{h}(B)$ where $h(B)$ is given by Eqs. (9) and (10) for the homogeneous (solid line and filled points) and wind density profile (long dashed line and open circles) as a function of the parameter $B$. The horizontal lines represent the scatter of the Ghirlanda correlation in the two scenarios (solid and long-dashed line respectively). The shaded region represents the $(1 \sigma)$ uncertainty on the parameter $B(=-1.08 \pm 0.17)$ found through the fit of the LZ05 correlation with the 18 GRBs.

\subsection{Wind density profiles}

If $t_{\mathrm{j}}$ is related to the semiaperture angle of the jet according to Eq. (6), the LZ05 correlation implies:

$E_{\gamma, \mathrm{iso}} \theta_{\mathrm{j}, \mathrm{w}}^{2} \sim E_{\gamma} \propto E_{\mathrm{p}}^{\prime A /(1-B)} \theta_{\mathrm{j}}^{2(B+1) /(1-B)}\left(n \eta_{\gamma}\right)^{-B /(1-B)}$.

For the same considerations reported in the previous section, we derive $B=-1$ if only the term $n \eta_{\gamma}$ contributes to the scatter of the Ghirlanda correlation in the wind density case (i.e. $\sigma \sim$ 0.08, Sect. 3.3 and Fig. 5). In this case the relation between the Ghirlanda correlation and the LZ05 correlation implies that $E_{\mathrm{p}}^{\prime} \propto E_{\gamma}^{2 / A}$.

If we consider that the scatter of the Ghirlanda correlation (long dashed horizontal line in Fig. 8) is entirely due to the term $\theta_{\mathrm{j}}^{2(B+1) /(1-B)}$ we can derive even shallower (with respect to the homogeneous case) constraints on the parameter $B \in(-1.45,-0.65)$ (Fig. 8 - long dashed curve). Again in the intermediate case, i.e. both $n \eta_{\gamma}$ and $\theta_{\mathrm{j}}$ contributing to the scatter of the Ghirlanda correlation, if we assume the range of possible values of $B$ resulting from the fit of the LZ05 correlation, then the term $\theta_{j}^{2(B+1) /(1-B)}$ contributes at most $\sim 60 \%$ of the scatter observed in the Ghirlanda correlation.

\section{The Ghirlanda correlation in the comoving frame}

The values of $E_{\mathrm{p}}^{\prime}$ and $E_{\gamma}$ we derive are the ones seen in the GRB rest frame but not in the fireball comoving frame.

In the simplest and standard scenario, which assumes that the observer's line of sight is within the jet opening angle and that the jet is homogeneous, $E_{\mathrm{p}}$ and $E_{\gamma}$ are both boosted by a factor $\sim 2 \Gamma$, i.e. the blueshift of the photons. 
Then the comoving $E_{\gamma}^{\mathrm{com}}=E_{\gamma} /(2 \Gamma)$ and $E_{\mathrm{p}}^{\mathrm{com}}=E_{\mathrm{p}}^{\prime} /(2 \Gamma)$. Here $\Gamma$ is the bulk Lorentz factor of the fireball emitting the prompt radiation.

We then exploit the fact that we see a very tight correlation (in the rest, but not comoving, frame) to pose some limits on the physics (i.e. the dynamics and the radiation process) of bursts.

Let us assume that the most general Ghirlanda correlation described by a generic power law, i.e. $E_{\mathrm{p}}^{\prime} \propto E_{\gamma}^{g}$, which then represents the correlation for both the homogeneous and wind density profiles. In the comoving frame, each point must be corrected by the bulk Lorentz factor of that burst. If these bulk Lorentz factors are uncorrelated with $E_{\gamma}$ or $E_{\mathrm{p}}^{\prime}$, then in the comoving frame the correlation is destroyed. It is unlikely that two quantities that are not correlated in the comoving frame are correlated in the rest frame. We then are obliged to assume that $\Gamma$ is a function of $E_{\gamma}$ (or, equivalently, of $E_{\gamma}^{\text {com }}$ ). To this aim, we assume a simple power law relation:

$$
\Gamma \propto\left(E_{\gamma}^{\mathrm{com}}\right)^{x} ; \quad \Gamma \propto E_{\gamma}^{x /(1+x)} .
$$

The two relations above are equivalent. In the comoving frame we have:

$E_{\mathrm{p}}^{\mathrm{com}} \propto\left(E_{\gamma}^{\mathrm{com}}\right)^{x g-x+g}$.

Note that:

- if $x=0$ (i.e. all bursts have the same Lorentz factor) then the slope in the comoving and in the rest frame is the same;

- if $g=1$ (i.e. wind case), the correlation is linear also in the comoving frame;

- the exponent $x$ can be thought of as determined by a particular dynamical model of the fireball. Once $x$ is fixed, then the exponent $(x g-x+g)$ appearing in Eq. (12) should be explained by the radiative process. Vice versa, if we have reason to fix, through a specific radiation model, the exponent of Eq. (12), then we have information on the dynamics of the fireball.

For the wind case (i.e. $g=1$ ) we obtain a linear relation, whose slope is therefore "Lorentz invariant". This of course would greatly help to explain the existence of the Ghirlanda correlation, since one of the main parameters, the bulk Lorentz factor, does not enter its determination. Thus, in the case of the wind, the explanation of the correlation should be found in the radiation process only, independently of the dynamics.

\section{Discussion}

We have shown that the model-independent correlation recently found by LZ05 between the isotropic emitted energy $E_{\gamma, \text { iso }}$, the rest frame peak energy $E_{\mathrm{p}}^{\prime}$ and the jet break time $t_{\mathrm{j}}^{\prime}$, calculated in the rest frame, is equivalent to the Ghirlanda correlation.

We have also shown that an even tighter correlation is found assuming that the circumburst density is distributed with an $r^{-2}$ wind profile. Remarkably enough, this Ghirlandawind correlation is linear, and this slope is independent of the Lorentz correction needed to find the correlation in the comoving frame.
We are aware that the wind-like distribution of the circumburst medium is not favored by the existing afterglow modeling, but the advantage of having a linear Ghirlanda correlation is sufficient to justify a deeper analysis, to determine if there are neglected effects that might hide the presence of the wind. This is beyond the scope of the present paper, and we defer this issue to future studies. Here we only mention that one of the main assumptions of the afterglow modeling might be particularly suspect, namely the hypothesis that the equipartition parameters $\epsilon_{B}$ and $\epsilon_{e}$ (i.e. the fraction of the dissipated energy converted in the magnetic field and in the electron energy, respectively) are kept fixed during the entire afterglow phase (while they have very different values from burst to burst). For instance, in the case of $\epsilon_{B} \propto \Gamma^{-\lambda}$, the synchrotron cooling is enhanced at later times with respect to the case of a constant $\epsilon_{B}$, and this makes the light curve of a fireball moving in a wind circumburst environment mimic the evolution of a fireball expanding in a uniform medium with a constant $\epsilon_{B}$ (the two cases becomes almost indistinguishable for $\lambda=2$ ). Consider also that all the evidence we have point towards a massive stellar progenitor of GRBs, and it is difficult to understand why there is no sign of wind around such massive stars. The fact that $\epsilon_{B}$ changes (as long as its evolution law does not change) produces a different decay law of the afterglow flux (with respect to a not-evolving $\epsilon_{B}$ ), but with no breaks. Therefore in this case $\epsilon_{B}$ does not enter in the estimate of the jet opening angle (as long as we are in the adiabatic regime).

The finding (Ghirlanda et al. 2005b) of a somewhat tighter and steeper Ghirlanda correlation in the wind density case than the homogenous case can be used to constrain the cosmological prameters similarly to what was done through the Ghirlanda correlation in the homogeneous case (Ghirlanda et al. 2004a; Firmani et al. 2005).

As pointed out by Ghirlanda et al. (2005a), the existence of the Ghirlanda correlation explains the Amati correlation between $E_{\gamma \text { iso }}$ and $E_{\mathrm{p}}^{\prime}$ (Amati et al. 2002; Ghirlanda et al. 2004b), and in particular explains why the Amati correlation has a much larger scatter than the Ghirlanda correlation. If GRBs are characterized by a distribution of semiaperture angles for each value of $E_{\mathrm{p}}^{\prime}$, then one sees a variety of $E_{\gamma \text {,iso-values for }}$ each value of $E_{\mathrm{p}}^{\prime}$. If the (observed) distribution of aperture angles has a preferred value, where it peaks, then this naturally produces a correlation in the $E_{\mathrm{p}}^{\prime}-E_{\gamma \text {,iso }}$ plane. Furthermore, it is conceivable that the bursts with spectroscopically measured redshifts are the brightest, hence with the smallest aperture angles (for a given redshift), hence lying at the large $E_{\gamma, \text { iso }}$ end of the real distribution. We have evidence that this is what is happening (Ghirlanda et al. 2005a).

Thus the Amati correlation can be easily explained by assuming the existence of i) the Ghirlanda correlation and ii) a (peaked) distribution of jet aperture angles. Therefore what remains to be explained is the Ghirlanda correlation itself which relates the intrinsic collimation corrected quantities. We have pointed out that there might be a difference between the apparent and the comoving Ghirlanda correlation, and from the theoretical point of view it is the comoving one that should be explained. As Rees \& Meszaros (2005) pointed out, a tight relation between the peak energy of the spectrum and the total 
emitted energy resembles a thermal process, where the peak energy is a measure of temperature. It is in this direction that we plan to investigate in the future.

The linear, wind-like, Ghirlanda correlation implies that the number of "relevant" photons (the ones with energies close to $E_{\mathrm{p}}^{\prime}$ ) is constant in all bursts, and approximately equal to $N_{\gamma}=10^{57}$, a number (coincidentally?) close to the number of baryons in one solar mass. In the "standard" scenario, in which the primary energy is injected close to the putative newly born black hole in a high entropy form, the requirement of a fixed number of photons $N_{\gamma}$ translates into the requirement that the product of the injected energy $E$ and the typical size of the injection region $R$ is constant. This can be seen in a simple way by noting that $a T^{4} R^{3} /(k T) \sim N_{\gamma}$, where $T$ is the temperature of the initial blackbody. Since $E \sim k T N_{\gamma}$, then $E R \propto N_{\gamma}^{4 / 3}=$ const. In the framework of the standard scenario, in the absence of an additional injection of energy, the number of photons is conserved during the acceleration and the coasting phase. Then, when the fireball becomes transparent, this blackbody-like component has a fixed number of photons. But bursts with different baryon loading would become transparent at different times, meaning that different fractions of the energy initially contained in the radiation field have survived the conversion to bulk kinetic energy. This means different efficiency factors $\eta_{\gamma}$, which is contrary to one of the main assumptions leading to the construction of the Ghirlanda correlation itself, which has been derived assuming the same $\eta_{\gamma}$ for all bursts.

If some extra dissipation of kinetic energy occurs after the acceleration phase, before the transparency radius, and the main radiation process is Comptonization, then we can reconvert part of the kinetic energy into radiation energy, leaving the number of photons unaltered. The available time to do it will be proportional to the transparency radius, so we have the positive feedback that sources that becomes transparent later (i.e. more baryon loaded, and hence with a radiation content that will be less energetic) have more possibilities to be re-energized by this putative dissipation (Rees \& Meszaros 2005). This positive feedback can narrow the range of radiative efficiencies. In any case, we face the problem to explain why bursts with the same number of photons have different total energies.

Apart from these theoretical considerations, there might be an "observational" way to see if the real $E_{\gamma}-E_{\mathrm{p}}^{\prime}$ is wind-like or constant density-like. Assume that there is a correlation between $E_{\mathrm{p}}$ and $E_{\gamma}$ and suppose that it will be possible to estimate the bulk Lorentz factor of the fireball associated to the prompt emission (that should be roughly equal to the bulk Lorentz factor of the very early afterglow, and controls the time at which the peak flux of the afterglow occurs). Preliminary attempts to estimate it have been made by e.g. Soderberg \& Ramirez-Ruiz (2002) for GRB 990123. Then, if by "de-beaming" the $E_{\gamma}$ and $E_{\mathrm{p}}^{\prime}$ values (i.e. computing them in the comoving frame) in the case of homogeneous density one finds an equally tight correlation, this would strongly support the homogeneous density hypothesis. Perhaps more importantly, one will also obtain the relation between $\Gamma$ and $E_{\gamma}^{\text {com }}$. On the contrary, if the de-beamed quantities (calculated in the case of a homogeneous density) do not correlate, this will strongly argue in favor of the wind hypothesis (in such a case the de-beamed quantities still correlate).

Acknowledgements. We thank the referee Amir Levinson for his valuable comments. We thank Annalisa Celotti and Davide Lazzati for discussions. The Italian MIUR and INAF are thanked for funding (Cofin grant 2003020775_002).

\section{References}

Amati, L., Frontera, F., Vietri, M., et al. 2000, Science, 290, 953

Amati, L., Frontera, F., Tavani, M., et al. 2002, A\&A, 390, 81

Amati, L. 2004 [arXiv:astro-ph/0405318]

Andersen, M. I., Masi, G., Jensen, B. L., \& Hjorth, J. 2003, GCN Circ., 1993

Atteia, J.-L., Kawai. N., Vanderspek, R., et al. 2005, ApJ, 626, 292

Atteia, J.-L. 2003, A\&A, 407, L1

Barraud, C., Olive, J.-F., Lestrade, J. P., et al. 2003, A\&A, 400, 1021

Barth, A. J., Sari, R., Cohen, M. H., et al. 2003, ApJ, 584, L47

Berger, E., Kulkarni, S. R., Bloom, J. S., et al. 2002, ApJ, 581, 981

Berger, E., Kulkarni, S. R., Pooley, G., et al. 2003, Nature, 426, 154

Bjornsson, G., Hjorth, J., Jakobsson, P., Christensen, L., \& Holland, S. 2001, ApJ, 552, L121

Bloom, J. S., Frail, D. A., \& Kulkarni, S. R. 2003, ApJ, 594, 674

Blustin, A. J., Band, D., Barthelmy, S., et al. 2005

[arXiv: astro-ph/0507515]

Chevalier, R. A., \& Li, Z. Y. 2000, ApJ, 536, 195

Della Valle, M., Malesani, D., Benetti, S., et al. 2003, A\&A, 406, L33

Djorgovsky, S. G., Kulkarni, S. R., Bloom, J. S., et al. 1998, ApJ, 508, L17

Djorgovsky, S. G., Frail, D. A., Kulkarni, S. R., et al. 2001, ApJ, 562, 654

Eichler, D., \& Levinson, A. 2004, ApJ, 614, L13

Firmani, C., Ghisellini, G., Ghirlanda, G., \& Avila-Reese, V. 2005, MNRAS, 630, L1

Foley, R. J., Chen, H.-W., Bloom, J., \& Prochaska, J. X. 2005, GCN Circ., 3483

Frail, D. A., Kulkarni, S. R., Sari, R., et al. 2001, ApJ, 562, L55

Frail, D. A., Soderberg, A. M., Kulkarni, S. R., et al. 2005, ApJ, 619, 994

Ghirlanda, G., Ghisellini, G., Lazzati, D., \& Firmani, C. 2004a, ApJ, 613, L13

Ghirlanda, G., Ghisellini, G., \& Lazzati, D. 2004b, ApJ, 616, 331

Ghirlanda, G., Ghisellini, G., \& Firmani, C. 2005a, MNRAS, 361, L10

Ghirlanda, G., Ghisellini, G., Firmani, C., Nava, L., \& Tavecchio, F. 2005b, A\&A, submitted

Greiner, J., Guenther, E., Klose, S., \& Schwarz, R. 2003a, GCN Circ., 1886

Greiner, J., Peimbert, M., Estaban, C., et al. 2003b, GCN Circ., 2020

Hjorth, J., Andersen, M. I., Cairos, L. M., et al. 1999, GCN Circ., 219

Hjorth, J., Moller, P., Gorosabel, J., et al. 2003, ApJ, 597, 699

Holland, S. T., Weidinger, M., Fynbo, J. P. U., et al. 2003, AJ, 125, 2291

Holland, S. T., Bersier, D., Bloom, J. S., et al. 2004, AJ, 128, 1955

Israel, G., Marconi, G., Covino, S., et al. 1999, A\&A, 384, L5

Jakobsson, S. P., Hjorth, J., Fynbo, J. P. U., et al. 2003, A\&A, 408, 941

Jakobsson, P., Hjorth, J., Fynbo, J. P. U., et al. 2004, A\&A, 427, 785

Jimenez, R., Band, D., \& Piran, T. 2001, ApJ, 561, 171

Klose, S., Greiner, J., Rau, A., et al. 2004, AJ, 128, 1942

Klotz, A., Boer, M., Atteia, J.-L., et al. 2005, A\&A, 439, L35

Kulkarni, S. R., Djorgovsky, S. G., Odewahn, S. C., et al. 1999, Nature, 398, 389 
Levinson, A., \& Eichler, D. 2005, ApJ, 629, L13

Liang, E., \& Zhang, B. 2005, ApJ, in press [arXiv: astro-ph/0504404]

Mirabal, N., Bonfield, D., \& Schawinski, K. 2005, GCN, 3488

Moller, P., Fynbo, J. P. U., Hjorth, J., et al. 2002, A\&A, 396, L21

Panaitescu, A., \& Kumar, P. 2000, ApJ, 543, 66

Panaitescu, A., \& Kumar, P. 2001, ApJ, 560, L49

Panaitescu, A., \& Kumar, P. 2002, ApJ, 571, 779

Press, W. H., et al. 1999, Numerical Recipes in C (Cambridge University Press), 661

Price, P. A., Kulkarni, S. R., Berger, E., et al. 2003, ApJ, 589, 838

Rees, M. J., \& Meszaros, P. 2005, ApJ, 628, 847

Rol, E., Vreeswijk, P., \& Jaunsen, A. 2003, GCN Circ., 1981
Sakamoto, T., Lamb, D. Q., Kawai, N., et al. 2005, ApJ, 629, 311 Sari, R. 1999, ApJ, 519, L17

Soderberg, A. M., \& Ramirez-Ruiz, E. 2002, MNRAS, 330, L24

Stanek, K. Z., Garnavich, P. M., Nutzman, P. A., et al. 2005, ApJ, 626, L5

Toma, K., Yamazaki, R., \& Nakamura, T. 2005, ApJ, 635, 481

Vanderspek, R., Sakamoto, T., Barraud, C., et al. 2004, ApJ, 617, 1251

Vreeswijk, P. M., Rol, E., Hjorth, J., et al. 1999, GCN Circ., 496

Vreeswijk, P. M., Fruchter, A., Kaper, L., et al. 2001, ApJ, 546, 672

Weidinger, M., Fynbo, J. P. U., Hjorth, J., et al. 2003, GCN Circ., 2215

Xu, D., ApJ, submitted [arXiv: astro-ph/0504052]

Yamazaki, R., Ioka, K., \& Nakamura, T. 2004, ApJ, 606, L33

Zeh, A., Klose, S., \& Kann, D. A. 2005, ApJ, preprint 